\title{
Arterial Dissection
}

National Cancer Institute

\section{Source}

National Cancer Institute. Arterial Dissection. NCI Thesaurus. Code C99704.

A tear within the wall of the artery. 